\title{
Linearity Test with Unit Root in TV-ESTAR Framework
}

\author{
Jinqi Song \\ Science and Technology College, Jiangxi Normal University, Nanchang, China
}

\begin{abstract}
Firstly, this paper proposes F statistic whose limit distribution and critical values are also provided to test nonlinearity and structure change with unit root in TV-ESTAR model framework. The results show that the distribution of $\mathrm{F}$ statistic is nonstandard. Then, this paper analyzes finite sample characteristics of $F$ statistics through the Monte Carlo simulation and founds F statistics has better power than kss statistics in Kapetanios et al to test nonlinear unit root with structure change.
\end{abstract}

Keywords: Time Varying Exponential Smooth Transition Autoregressive Model; Structure Change; Unit Root; Linearity Test.

\section{Literature Review}

Since the 1980s, a large number of nonlinear measurement models, methods and technologies have emerged. Due to the heterogeneity of economic agent, the structure changes are smooth rather than sudden, the Smooth Transition Autoregressive (STAR) model, STAR model has been recognized by the economics community and become one of the most active models. In the linear test under the unit root condition, one type of research uses the lag of the variable or its difference as the conversion variable to explore nonlinearity, to reflect that economic agent make different choices with the change of historical information, and the other type of research Then choose time $t$ as the conversion variable to analyze the nonlinearity, to reflect the nonlinear characteristics of the structural changes in the behavior of economic agents over time.

Kapetanios et al (2003, hereinafter referred to as kss) proposed a unit root linear test method within the framework of the exponentially smoothed autoregressive (ESTAR) model.

Kilic (2004) pointed out that the stationarity of the sequence should be considered in the linear test of the STAR model. Sandberg (2008) assumes $z_{t}=\mathrm{t}$, he discussedthe linear test of TV-LSTAR with $\mathrm{t}$ and $y_{t-1}$ as Transfer variable.

Through the first-order Taylor expansion, it is found that the Wald linear test statistic is nonstandard Brownian motion. The simulation results show that when the sequence is a random walk process, its critical value is higher than that of the standard chi-square distribution under stationary conditions, but smaller than Kilic (2004). The simulation experiment also shows that when the linear test is performed on the sequence whose data generation process is the unit root, the rejection frequency of Sandberg (2008) and Killic (2004) are both higher than the given nominal 5\% significance level, but Kilic (2004)) Has a higher rejection frequency. Therefore, using the standard chi-square distribution may get wrong conclusions.

Based on kss (2003), Kilic (2011) uses $\Delta \mathrm{y}_{\mathrm{t}-1}$ as the conversion variable, and proposes a method to convert the parameter space through a search mechanism to find the smallest $t_{\mathrm{ESTAR}}$ for ESTAR Model unit root test and linearity test.

The above literature shows that there may be both nonlinearity (kss, 2003; Kilic, 2004, 2011) and structural changes (Sandberg, 2008) in the unit root linear test. Campbell and Perron (1991) pointed out that if the deterministic trend term is set incorrectly, it will seriously affect the power of the ADF test. The unit root test under the ESTAR framework proposed by Kss (2003), after rejecting the null hypothesis, implicitly believes that under the alternative hypothesis, although the mean recovery speed of different deviations changes nonlinearly, the same deviation from the mean recovery speed at any time Unchanged, that is, the unit root test and linearity test are performed under the assumption that there is no structural change. Sandberg (2008) considered time $t$ as the conversion variable, but only pointed out that when the sequence is a unit root process, the standard chi-square distribution cannot be used, and did not study the relative effectiveness of the unit root linear test under the STAR framework and the TV-STAR framework. 
The ESTAR model is a very useful model. In this paper, structural changes are added based on kss (2003), and the TV-ESTAR model framework is constructed to analyze the nonlinearity and structural changes in the unit root linear test. The article is arranged as follows: The second part gives the TVESTAR model and the null hypothesis for testing whether the unit root has nonlinearity and structural changes; the third part introduces the TV-ESTAR model unit root linearity test method and derives the test statistic $F$ And the limit distribution of $F_{-} s$, and the critical value of the test statistic is simulated at the same time; the fourth part simulates the level and power of the statistic through the Monte Carlo experiment, and compares it with the linear test power of the kss statistic without structural changes, the fifth part is the conclusion.

\section{TV-ESTAR Model and Assumptions}

Assuming that the variables are non-stationary, the non-linearity and structure change tests are performed in the STAR model with structural changes, and TV-ESTAR formula (1) is given:

$$
\Delta y_{t}=\rho_{1} y_{t-1} G\left(\gamma_{1}, y_{t-d}\right)\left(1-S\left(\gamma_{2}, t, c\right)\right)+\rho_{2} y_{t-1} G\left(\gamma_{1}, y_{t-d}\right) S\left(\gamma_{2}, t, c\right)+\varepsilon_{t}
$$

$G\left(\gamma_{1}, y_{t-1}\right)=1-\exp \left(-\gamma_{1} y_{t-1}^{2}\right)$, In order to facilitate the construction of statistics for structural changes and nonlinear tests, assume $S\left(\gamma_{2}, t, c\right)=\left(1+\exp \left(-\gamma_{2}(t-c)\right)\right)^{-1}-0.5, \gamma_{2}>0$, The null hypothesis for testing whether there is nonlinearity can be set to $\gamma_{1}=0$, and the null hypothesis for testing whether there is a structural change is $\gamma_{2}=0$ and is unrecognizable, the first-order Taylor expansion can be used to obtain equation (2):

$$
\Delta y_{t}=\beta_{1} y_{t-1}^{3}+\beta_{2} t y_{t-1}^{3}+R\left(\gamma_{1}, \gamma_{2}\right)+\varepsilon_{t}
$$

$\beta_{1}=\rho_{2} \gamma_{1}+\frac{1}{4}\left(\rho_{2}-\rho_{1}\right) \gamma_{1} \gamma_{2} c, \beta_{2}=\frac{1}{4}\left(\rho_{1}-\rho_{2}\right) \gamma_{1} \gamma_{2}, \mathrm{R}\left(\gamma_{1}, \gamma_{2}\right)$ Is the remainder after Taylor expansion, its value is 0 under the condition that the null hypothesis is established, and it does not affect the asymptotic distribution. According to formula (2), the null hypothesis of linearity test and structural change becomes $\mathrm{H} 0: \beta_{1}=\beta_{2}=0, \mathrm{H} 01: \beta_{2}=0$. Not rejecting $\mathrm{H} 0$, the sequence does not have nonlinearity, it is a linear unit root process. After rejecting $\mathrm{H} 0$, then rejecting $\mathrm{H} 01$, the model has both nonlinearity and structural mutation, the TV-ESTAR model is estimated. After rejecting H0, accepting H01, the model has only nonlinearity, and there is no structural change, which is the ESTAR model. If there is a sequence correlation, like kss (2003), equation (1) can be extended to obtain a higher-order test equation (3):

$$
\Delta y_{t}=\beta_{1} y_{t-1}^{3}+\beta_{2} t y_{t-1}^{3}+\sum_{i=1}^{p} \kappa_{i} \Delta y_{t-i}+R\left(\gamma_{1}, \gamma_{2}\right)+\varepsilon_{t}
$$

\section{Test Statistics for Unit Root Linearity and Structural Change}

To test $\mathrm{H} 0$, assume $\widehat{\boldsymbol{\beta}}=\left(\hat{\beta}_{1}, \hat{\beta}_{2}\right)^{\prime}$ is ols of $(5), \boldsymbol{x}_{\boldsymbol{t}}=\left(y_{\mathrm{t}-1}^{3}, t y_{\mathrm{t}-1}^{3}\right)^{\prime}$, then:

$$
\widehat{\beta}-\beta=\left(\sum x_{t} x_{t}^{\prime}\right)^{-1} \sum x_{t} \varepsilon_{t}
$$

Construct the F statistic as follows (Hamilton, 1999):

$$
\mathrm{F}=(\boldsymbol{R}(\widehat{\boldsymbol{\beta}}-\boldsymbol{\beta}))^{\prime}\left(\widehat{\mathrm{s}}^{2} \boldsymbol{R}\left(\sum \boldsymbol{x}_{\boldsymbol{t}} \boldsymbol{x}_{\boldsymbol{t}}^{\prime}\right)^{-1} \boldsymbol{R}^{\prime}\right)^{-1}(\boldsymbol{R}(\widehat{\boldsymbol{\beta}}-\boldsymbol{\beta})) / m
$$

$\widehat{\mathrm{s}}^{2}$ Is the residual variance estimated by the model's least squares, $\boldsymbol{R}$ is $2 \times 2$ Identity matrix, $m=$ $2, \boldsymbol{R} \boldsymbol{\beta}=\boldsymbol{r}, \boldsymbol{r}=(0,0)^{\prime}$, assume $\boldsymbol{\Gamma}=\operatorname{diag}\left(\mathrm{T}^{2}, \mathrm{~T}^{3}\right)$, we get: 
Volume 13 (2021)

$$
\begin{gathered}
\boldsymbol{\Gamma}(\widehat{\boldsymbol{\beta}}-\boldsymbol{\beta})=\left(\boldsymbol{\Gamma}^{-1}\left(\sum \boldsymbol{x}_{\boldsymbol{t}} \boldsymbol{x}_{\boldsymbol{t}}{ }^{\prime}\right) \boldsymbol{\Gamma}^{-1}\right)^{-1} \boldsymbol{\Gamma}^{-1} \sum \boldsymbol{x}_{\boldsymbol{t}} \varepsilon_{t} \\
\boldsymbol{\Gamma}^{-1}\left(\sum \boldsymbol{x}_{\boldsymbol{t}} \boldsymbol{x}_{\boldsymbol{t}}{ }^{\prime}\right) \boldsymbol{\Gamma}^{-1}=\left[\begin{array}{cc}
\mathrm{T}^{-4} \sum y_{\mathrm{t}-1}^{6} & \mathrm{~T}^{-5} \sum t y_{\mathrm{t}-1}^{6} \\
\mathrm{~T}^{-5} \sum t y_{\mathrm{t}-1}^{6} & \mathrm{~T}^{-6} \sum t^{2} y_{t-1}^{6}
\end{array}\right] \\
\left\{\boldsymbol{\Gamma}^{-1} \sum \boldsymbol{x}_{\boldsymbol{t}} \varepsilon_{\mathrm{t}}\right\}^{\prime}=\left[\begin{array}{ll}
\mathrm{T}^{-2} \sum y_{t-1}^{3} \varepsilon_{t} & \mathrm{~T}^{-3} \sum t y_{t-1}^{3} \varepsilon_{t}
\end{array}\right]
\end{gathered}
$$

According to the central limit theorem of functionals and the continuous mapping theorem (Hamilton, 1999), there is ( $\Rightarrow$ means weak convergence in distribution)

$$
\begin{gathered}
\boldsymbol{\Gamma}^{-1}\left(\sum \boldsymbol{x}_{\boldsymbol{t}} \boldsymbol{x}_{\boldsymbol{t}}{ }^{\prime}\right) \Gamma^{-1} \Rightarrow \sigma^{6}\left[\begin{array}{ll}
\int_{0}^{1} \mathrm{~W}(\mathrm{r})^{6} \mathrm{dr} & \int_{0}^{1} \mathrm{rW}(\mathrm{r})^{6} \mathrm{dr} \\
\int_{0}^{1} \mathrm{rW}(\mathrm{r})^{6} \mathrm{dr} & \int_{0}^{1} \mathrm{r}^{2} \mathrm{~W}(\mathrm{r})^{6} \mathrm{dr}
\end{array}\right] \equiv \sigma^{6} \boldsymbol{Q} \\
\left\{\boldsymbol{\Gamma}^{-1} \sum \boldsymbol{x}_{\boldsymbol{t}} \varepsilon_{\mathrm{t}}\right\}^{\prime} \Rightarrow \sigma^{4}\left[\int_{0}^{1} \mathrm{~W}(\mathrm{r})^{3} \mathrm{dW}(\mathrm{r}) \quad \int_{0}^{1} \mathrm{rW}(\mathrm{r})^{3} \mathrm{dW}(\mathrm{r})\right] \equiv \sigma^{4} \boldsymbol{h}^{\prime} \\
\boldsymbol{\Gamma}(\widehat{\boldsymbol{\beta}}-\boldsymbol{\beta}) \Rightarrow \sigma^{-2} \boldsymbol{Q}^{-1} \boldsymbol{h}
\end{gathered}
$$

under $\mathrm{H} 0, \hat{\mathrm{s}}^{2} \stackrel{\mathrm{p}}{\rightarrow} \sigma^{2}$, Thus the F statistic can become:

$$
\begin{array}{r}
\mathrm{F}=(\boldsymbol{R} \boldsymbol{\Gamma}(\widehat{\boldsymbol{\beta}}-\boldsymbol{\beta}))^{\prime}\left(\sigma^{2} \boldsymbol{R} \boldsymbol{\Gamma}\left(\sum \boldsymbol{x}_{\boldsymbol{t}} \boldsymbol{x}_{\boldsymbol{t}}\right)^{-1} \boldsymbol{\Gamma} \boldsymbol{R}^{\prime}\right)^{-1}(\boldsymbol{R} \boldsymbol{\Gamma}(\widehat{\boldsymbol{\beta}}-\boldsymbol{\beta})) / 2 \\
\Rightarrow\left(\boldsymbol{R} \sigma^{-2} \boldsymbol{Q}^{-1} \boldsymbol{h}\right)^{\prime}\left(\sigma^{2} \boldsymbol{R}\left(\sigma^{6} \boldsymbol{Q}\right)^{-1} \boldsymbol{R}^{\prime}\right)^{-1}\left(\boldsymbol{R} \sigma^{-2} \boldsymbol{Q}^{-1} \boldsymbol{h}\right) / 2=\boldsymbol{h}^{\prime} \boldsymbol{Q}^{-1} \boldsymbol{h} / 2
\end{array}
$$

Therefore, the distribution of the F statistic of the unit root linear test in the TV-ESTAR model is obtained. For the H01 test, let $\boldsymbol{R}=(0,1), m=1, \boldsymbol{R} \boldsymbol{\beta}=\mathrm{r}, \mathrm{r}=0, \boldsymbol{\Gamma}=\operatorname{diag}\left(\mathrm{T}^{2}, \mathrm{~T}^{3}\right)$, After calculating we get

$$
F_{S} \Rightarrow \frac{\left\{\int_{0}^{1} W(r)^{6} d r \int_{0}^{1} r W(r)^{3} d W(r)-\int_{0}^{1} r W(r)^{6} d r \int_{0}^{1} W(r)^{3} d W(r)\right\}^{2}}{\int_{0}^{1} W(r)^{6} d r\left\{\int_{0}^{1} W(r)^{6} d r\left(\int_{0}^{1} r^{2} W(r)^{6} d r\right)-\left(\int_{0}^{1} r W(r)^{6} d r\right)^{2}\right\}}
$$

The above two test statistics both converge to the random functional of the Wiener process. For the case of non-zero mean and deterministic trend terms, the asymptotic distribution of the test statistics is the same, but instead of using $\mathrm{W}(r) \mathrm{W}(r)^{*}$ is used, where $\mathrm{W}(r)^{*}$ is the Brownian motion of de-mean and de-trend respectively (kss, 2003). For the equation (3) with sequence correlation, its limit distribution has not changed. Therefore, the critical value of the $\mathrm{F}$ statistic with sequence correlation will not change. Using Monte Carlo simulation test (50,000 times) to obtain F, the critical value of $F_{-}$s statistic is shown in Table 1 and Table 2 .

Table 1. F test critical value

\begin{tabular}{|c|c|c|c|c|c|c|c|c|c|}
\hline \multirow{2}{*}{$\mathrm{T}$} & \multicolumn{3}{|c|}{ Case 1 } & \multicolumn{3}{|c|}{ Case 2 } & \multicolumn{3}{c|}{ Case 3 } \\
\cline { 2 - 10 } & $10 \%$ & $5 \%$ & $1 \%$ & $10 \%$ & $5 \%$ & $1 \%$ & $10 \%$ & $5 \%$ & $1 \%$ \\
\hline 50 & 2.94 & 3.75 & 5.74 & 4.88 & 5.98 & 8.60 & 5.97 & 7.07 & 9.76 \\
\hline 100 & 2.98 & 3.74 & 5.51 & 4.85 & 5.82 & 8.07 & 5.92 & 7.02 & 9.38 \\
\hline 200 & 2.95 & 3.67 & 5.35 & 4.80 & 5.76 & 7.84 & 5.92 & 7.00 & 9.30 \\
\hline$\infty$ & 2.95 & 3.65 & 5.31 & 4.82 & 5.73 & 7.82 & 5.88 & 6.96 & 9.33 \\
\hline
\end{tabular}

Note: Case 1, Case2, Case3 correspond to zero mean, non-zero mean, non-zero mean and deterministic trend, respectively. 
Table 2. $\mathrm{F}_{\mathrm{s}}$ test critical value

\begin{tabular}{|c|c|c|c|c|c|c|c|c|c|}
\hline \multirow{2}{*}{$\mathrm{T}$} & \multicolumn{3}{|c|}{ Case 1 } & \multicolumn{3}{c|}{ Case 2 } & \multicolumn{3}{c|}{ Case 3 } \\
\cline { 2 - 10 } & $10 \%$ & $5 \%$ & $1 \%$ & $10 \%$ & $5 \%$ & $1 \%$ & $10 \%$ & $5 \%$ & $1 \%$ \\
\hline 50 & 3.58 & 5.03 & 8.68 & 4.92 & 6.77 & 11.25 & 5.43 & 7.47 & 12.13 \\
\hline 100 & 3.59 & 5.06 & 8.37 & 4.96 & 6.70 & 11.15 & 5.40 & 7.43 & 12.09 \\
\hline 200 & 3.53 & 4.95 & 8.19 & 4.90 & 6.64 & 10.60 & 5.42 & 7.44 & 12.14 \\
\hline$\infty$ & 3.39 & 4.72 & 7.83 & 4.86 & 6.62 & 10.54 & 5.42 & 7.45 & 11.81 \\
\hline
\end{tabular}

Note: Same as Table 1

\section{The Level and Power of Test Statistics}

\subsection{Limited Sample Size Test}

First, briefly examine the finite sample characteristics of the F statistics Case 1, Case 2, and Case 3. The data generation process is as shown in equation (18):

$$
y_{t}=y_{t-1}+\varepsilon_{t}, \quad \varepsilon_{t}=\rho \varepsilon_{t-1}+\mu_{t}, t=1,2, \ldots, \mathrm{T}
$$

$\mu_{\mathrm{t}} \sim \operatorname{iid}(0,1), \rho=\{-0.5,0,0.5\}$, When the sample size is $\mathrm{T}=50,100$, and 200 , repeat 10,000 times. When calculating the value of the F test statistic, the test models under different settings include the first-order lag of $\Delta y_{t}$. Therefore, for the case of $\rho=0$, the test model is set too much, and the case of $\rho=-0.5,0.5$ is tested The model is correctly set, the nominal significance level is set to $5 \%$, and the test results are shown in Table 3 . It can be seen that in the process of generating these data, as the sample size increases, the horizontal distortion is smaller.

Table 3. Size test

\begin{tabular}{|c|c|c|c|c|c|c|c|c|c|}
\hline & \multicolumn{3}{|c|}{$\mathrm{T}=50$} & \multicolumn{3}{c|}{$\mathrm{T}=100$} & \multicolumn{3}{c|}{$\mathrm{T}=200$} \\
\cline { 2 - 11 } & Case1 & Case2 & Case3 & Case1 & Case2 & Case3 & Case1 & Case2 & Case3 \\
\hline$\rho=-0.5$ & 0.0374 & 0.0393 & 0.0319 & 0.0376 & 0.0409 & 0.0407 & 0.0439 & 0.0492 & 0.0421 \\
\hline$\rho=0$ & 0.048 & 0.0535 & 0.0546 & 0.0457 & 0.0507 & 0.0483 & 0.0493 & 0.0493 & 0.0512 \\
\hline$\rho=0.5$ & 0.058 & 0.0673 & 0.0771 & 0.0503 & 0.0565 & 0.0632 & 0.0485 & 0.0531 & 0.0542 \\
\hline
\end{tabular}

\subsection{Limited Sample Power Test}

For the sake of simplicity, and because non-zero mean (no deterministic trend) is a common situation in practical applications, we only list the results of the simulation of case 2 . The sample size is $\mathrm{T}=100$ and $\mathrm{T}=200$, and the nominal significance level is set to $5 \%$. Assuming that the structural change position parameter is in the middle of the sample point, the data generation process uses the TV-ESTAR formula (4), and different parameters are selected Value, take values for each group of parameters, repeat the simulation 10000 times, calculate the proportion of the rejection of the null hypothesis, that is, the rejection frequency. Since the data generation process is a non-linear model with structural changes, the higher the rejection frequency, Which indicates that the test is more effective, and for the sake of comparison, the rejection frequency obtained by using the kss test as a judgment is given at the same time.

let $\gamma_{2}=1, \gamma_{1}=\{0.1,0.5,1$, the combination of autoregressive parameters $\rho 1$ and $\rho 2$ is as follows $\rho_{1}=\{-0.05,-0.1,-0.3\}, \rho_{2}=\{-0.05,-0.1,-0.5,-0.9\}, \mathrm{T}=100, \mathrm{c}=50$.

From the simulation results (Table 4), it can be seen that when the given $\gamma_{1}$ is constant, the absolute value of $\rho_{1}, \rho_{2}$ is constantly increasing, and the power of all test statistics is constantly increasing, because this reflects the return of the sequence to the mean. The speed is getting faster and faster. When the absolute difference between the two parameters reflecting the structural change $\left|\rho_{1}-\rho_{2}\right|$ is small, that is, the structural change does not exist or is not obvious, the power of the $\mathrm{F}$ test 
of the linear test is lower than the power of the kss test, and the difference of $\left|\rho_{1}-\rho_{2}\right|$ keeps increasing, that is, the structure changes are becoming more and more obvious, and the power of $\mathrm{F}$ test is obviously better than that of kss test. For example, when $\gamma_{1}=1, \rho_{1}=-0.05$, and $\rho_{2}=-0.9$, the power of $\mathrm{F}$ statistic is $45 \%$ higher than that of kss statistic. The reason may be that the calculation of the $\mathrm{F}$ statistic requires more parameters to be estimated, and it is carried out under the assumption that there is a structural change, which will cause overestimation problems and reduce the test power, but when the structural change becomes more obvious, its advantages are obtained. Make up for the loss of efficacy. Given $\left|\rho_{1}-\rho_{2}\right|$, when the difference is constant, with the increase of the nonlinear conversion parameter $\gamma_{1}$, the power of both the kss test and the $F$ test increase, but the power of the $F$ test increases faster, and the power of the kss test increases more than the reason may be that the kss test is performed under the assumption that there is no structural change. The increase of $\gamma_{1}$ is equal to the relative increase of $\left|\rho_{1}-\rho_{2}\right|$ difference, so the power of the F statistic is improved.

Table 4. Power test $(\mathrm{T}=100)$

\begin{tabular}{|c|c|c|c|c|c|c|c|c|c|c|}
\hline \multicolumn{9}{|c|}{} & \multicolumn{3}{|c|}{$\gamma_{1}=0.1$} & \multicolumn{3}{c|}{$\gamma_{1}=0.5$} & \multicolumn{3}{c|}{$\gamma_{1}=1$} \\
\hline$\rho_{1}$ & $\rho_{2}$ & $\mathrm{~F}$ & $\mathrm{kss}$ & $\mathrm{F}_{\mathrm{S}}$ & $\mathrm{F}$ & $\mathrm{kss}$ & $\mathrm{F}_{\mathrm{S}}$ & $\mathrm{F}$ & $\mathrm{kss}$ & $\mathrm{F}_{\mathrm{S}}$ \\
\hline-0.05 & -0.05 & 0.1356 & 0.2153 & 0.0407 & 0.165 & 0.2445 & 0.0456 & 0.1767 & 0.2523 & 0.0486 \\
\hline-0.05 & -0.1 & 0.1848 & 0.3081 & 0.035 & 0.2429 & 0.3465 & 0.0472 & 0.2504 & 0.3469 & 0.0545 \\
\hline-0.05 & -0.5 & 0.4747 & 0.3758 & 0.1934 & 0.5379 & 0.3724 & 0.3037 & 0.504 & 0.3372 & 0.3067 \\
\hline-0.05 & -0.9 & 0.5457 & 0.3474 & 0.3553 & 0.5732 & 0.3317 & 0.4809 & 0.5573 & 0.3048 & 0.4779 \\
\hline-0.1 & -0.05 & 0.232 & 0.3345 & 0.06 & 0.3042 & 0.3805 & 0.0856 & 0.3107 & 0.3827 & 0.0798 \\
\hline-0.1 & -0.1 & 0.3003 & 0.4813 & 0.0364 & 0.4082 & 0.5437 & 0.0561 & 0.4108 & 0.5373 & 0.0636 \\
\hline-0.1 & -0.5 & 0.6575 & 0.5957 & 0.1892 & 0.7411 & 0.6232 & 0.3373 & 0.7299 & 0.6004 & 0.3474 \\
\hline-0.1 & -0.9 & 0.7195 & 0.5713 & 0.3868 & 0.7863 & 0.5816 & 0.5994 & 0.77 & 0.5485 & 0.6087 \\
\hline-0.3 & -0.05 & 0.558 & 0.4803 & 0.1871 & 0.6021 & 0.4513 & 0.2574 & 0.5974 & 0.4443 & 0.267 \\
\hline-0.3 & -0.1 & 0.6595 & 0.6822 & 0.1246 & 0.7702 & 0.6941 & 0.2215 & 0.7395 & 0.6807 & 0.217 \\
\hline-0.3 & -0.5 & 0.9495 & 0.9747 & 0.0732 & 0.9865 & 0.9881 & 0.1561 & 0.9844 & 0.9819 & 0.1803 \\
\hline-0.3 & -0.9 & 0.9695 & 0.9688 & 0.2662 & 0.9931 & 0.9858 & 0.5374 & 0.9903 & 0.9812 & 0.5723 \\
\hline
\end{tabular}

In general, if the model has obvious structural changes and nonlinearities, the F-test has better power than the kss test. When there are no structural changes and very weak nonlinearities, the F-test is inferior to the kss test. However, theF $F_{S}$ test is in this way Under the circumstances, it seems that the structure change can be judged more accurately. Therefore, in practical applications, starting from the linear model, there are two path options: 1) Perform the kss test first, and then perform the $\mathrm{F}_{\mathrm{S}}$ test, which is equivalent to special to general construction. Modular mode; 2) F-test first, then F_S test, which is equivalent to a special to general and then a special modeling method. No matter which path, $F_{S}$ must be checked. You can choose according to the following steps. $F_{S}$ is calculated under the framework of the TV-ESTAR model. If $\mathrm{H} 01$ is accepted, it means that there is no structural change in the model. Use kss for linearity test. If $\mathrm{H} 01$ is rejected, It is more accurate to use $\mathrm{F}$ statistic for linearity test.

\section{Conclusion}

This article discusses the linear test problem when the sequence is a unit root process under the framework of the TV-ESTAR model. The structure change is added on the basis of kss (2003), which allows the same degree of deviation from the mean value of different periods to recover at different speeds. The modeling process requires a two-stage test of the model, that is, first test the existence of nonlinearity, and then test the existence of structural changes after rejecting the null hypothesis of linear unit roots. Different from the kss test, the F statistic is given to test nonlinearity and structural changes based on the first-order Taylor expansion, and their limit distribution is derived. Since the 
limit distribution converges to the random functional of the Wiener process, the simulation gives The critical value of the test statistic. For the case of non-zero mean and trend terms, the same method of kss is used to remove the mean and the trend, and the critical value is also given. If there is a highorder serial correlation, the limit distribution of the test statistic does not change, so the critical value Still applicable.

Using the Monte Carlo test to simulate the limited sample characteristics of the proposed test statistic, it is found that when the data generation process is a nonlinear ESTAR model with structural changes, the power of the $\mathrm{F}$ test is better than that of the kss linear test.

\section{Acknowledgments}

Jiangxi Humanities and Social Sciences Fund Project "Non-stationary STR Model and Application Research" (TJ1505), Jiangxi Provincial Department of Education Science and Technology Research Project "Improving Jiangxi's Opening to the Outside World and FDI's New Advantages and Countermeasures" (GJJ171476).

\section{References}

[1] J Y Campbell, and P Perron (1991). Pitfalls and opportunities: What macroeconomists should know about unit roots, in NBER Macroeconomic Annual edited by O Blanchard and S Fischer. Cambridge. Massachusetts. The MIT Press, 1991:141-201.

[2] G Kapetanios, Y Shin and A Snell.Testing for a unit root in the nonlinear STAR framework[J]. Journal of Econometrics, 2003(112):359-379.

[3] Rehim Kilic. Linearity tests and stationarity[J]. Econometrics Journal,2004(7):55-62.

[4] Rehim Kilic. Test for a unit root in a stationary ESTAR process[J]. Econometrics Reviews, 2011(3):274302.

[5] Robinson Kruse. A new unit root test against ESTAR based on a class of modified statistics [J]. Statistical Papers, 2011(1):71-85.

[6] S Lundbergh, T Teräsvirta and V D Dijk.Time-varying smooth transition autoregressive models[J]. Journal of Business and Economic Statistics,2003(21):104-121.

[7] R Sandberg.Critical values for linearity tests in time-varying smooth transition autoregressive models when data are highly persistent [J]. Econometrics Journal,2008(11):638-647. 